\title{
SIGNATURES OF COHERENT SYSTEMS BUILT WITH SEPARATE MODULES
}

\author{
ILYA GERTSBAKH, ${ }^{*}$ Ben Gurion University \\ YOSEPH SHPUNGIN, ${ }^{* *}$ Shamoon College of Engineering \\ FABIO SPIZZICHINO, ${ }^{* * *}$ Sapienza University of Rome
}

\begin{abstract}
The signature is an important structural characteristic of a coherent system. Its computation, however, is often rather involved and complex. We analyze several cases where this complexity can be considerably reduced. These are the cases when a 'large' coherent system is obtained as a series, parallel, or recurrent structure built from 'small' modules with known signature. Corresponding formulae can be obtained in terms of cumulative notions of signatures. An algebraic closure property of families of homogeneous polynomials plays a substantial role in our derivations.
\end{abstract}

Keywords: Cumulative and tail signatures; recurrent; series; parallel system; anchor; homogeneous polynomial

2010 Mathematics Subject Classification: Primary 60K10

Secondary $90 \mathrm{~B} 25$

\section{Introduction}

In the analysis of coherent systems, the concept of signature is more and more revealing as a quite natural and very powerful tool, and different types of related application have been pointed out. The literature on this subject is extensive, and focuses on both conceptual and computational aspects. Some relevant references are presented in the bibliography; see in particular the monographs of [9] and [19], and the references therein.

An important and still challenging problem in this field is the computation of the signature for coherent systems of large order; in fact, rather complex computations are typically involved in such a task. Different types of method and of solution to this problem can be considered, depending on special system structures. In [22], in particular, a generating function approach was applied to the signature of linear consecutive $k$-out-of- $n$ systems. In this paper we show how the complexity can be sensibly reduced in the case of systems with other general structures. Namely, we consider systems that are coherent connections of different modules, which comprise separated sets of components.

We start by considering systems that can be decomposed into series or parallel connections of two subsystems. More precisely, starting from the signatures of two coherent systems $\delta_{1}$ and $s_{2}$, we study the signature of the 'super-system' $\&$ obtained as a series or as a parallel of $\delta_{1}$ and $s_{2}$. Thereafter we consider the case of recurrent systems, i.e. those systems that are

Received 6 January 2011; revision received 6 May 2011.

* Postal address: Shai Agnon 30/25, 69362 Tel-Aviv, Israel. Email address: elyager@ bezeqint.net

** Postal address: Department of Software Engineering, Shamoon College of Engineering, Beer Sheva, Bialik/Basel Streets, Beer Sheva, Israel. Email address: yosefs@sce.ac.il

*** Postal address: Department of Mathematics, Sapienza University of Rome, Piazzale Aldo Moro 2, 00185 Rome, Italy. Email address: fabio.spizzichino@uniroma1.it 
obtained as connections of several identical subsystems organized into a structure with known signature.

We shall see (Propositions 2 and 3 in Section 3, and Proposition 4 in Section 4) that solutions to our problem are obtained in terms of cumulative notions of signatures, rather than in terms of the standard notion of signature. Actually, the interest and the meaning of such notions have already been pointed out in the literature (see, in particular, [2] and [19, Chapters 5, 6]; see also [9, Chapters 6, 8]).

It will be important for our purposes to keep in mind that two different but equivalent definitions of signature can be given: one of probabilistic type and one which is purely combinatorial. The arguments used in the literature are based on the latter or the former type of definition, according to what is more convenient in the case at hand. In our problem we can use either of the two methods and it is convenient to rely on both of them in order to better illustrate our arguments and results.

The paper is organized as follows. In Section 2 we initially fix the notation and recall some basic definitions and facts related to the concept of signature. Then we point out relevant aspects of the 'cumulative' notions of signature. In Section 3 we describe some deductions concerning systems obtained as series or parallel connections of two systems. More precisely, we show how Proposition 2 (or the analogous Proposition 3) can be immediately proved by elementary algebraic arguments based on the 'probabilistic' definition of signature; then we give a heuristic interpretation in terms of the 'combinatorial' definition. We end this section with some comments of potential interest. In Section 4 we consider the case of recurrent systems; we shall see how the formulae obtained in Section 2 can be extended to this case. Finally, we devote Section 5 to the presentation of specific examples that illustrate the meaning and the use of our results. Some computational aspects are also sketched therein.

\section{Notation, definitions, and basic facts}

Consider the lifetime $T$ of a (binary) coherent system $\&$ (see, e.g. [1, Chapter 1]) formed with $n$ binary components $C_{1}, \ldots, C_{n}$. Let $\varphi_{s}:\{0,1\}^{n} \rightarrow\{0,1\}$ denote the corresponding structure function. We assume that $T_{1}, \ldots, T_{n}$, the lifetimes of $C_{1}, \ldots, C_{n}$, are independent with a common, continuous distribution function $F$. For the corresponding order statistics $T_{(1)}, \ldots, T_{(n)}$, it then holds that

$$
\mathrm{P}\left(T_{(1)}<\cdots<T_{(n)}\right)=1 .
$$

The system $\&$ (since it is coherent) will fail, with probability 1 , in concomitance with the failure of one and only one of the system's components, i.e. we can write

$$
\mathrm{P}\left(T=T_{(k)} \text { for some } k\right)=1 .
$$

The vector $s \equiv\left(s_{1}, \ldots, s_{n}\right)$, with

$$
s_{k}:=\mathrm{P}\left(T=T_{(k)}\right), \quad k=1,2, \ldots, n,
$$

is called the signature of $\&$ (see [18]). Then the signature of $\&$ is a specific probability distribution over $\{1, \ldots, n\}$. As pointed out in [18], we have the following very simple, but important, fact.

Proposition 1. The vector $s \equiv\left(s_{1}, \ldots, s_{n}\right)$ does not depend on the choice of $F$.

Thus, $s$ is a mere characteristic of the structure function $\varphi_{\S}$ of the system $\&$. In the case when $T_{1}, \ldots, T_{n}$ are independent and identically distributed (i.i.d.) we also find that the partition 
$\left\{\left(T=T_{(k)}\right), k=1,2, \ldots, n\right\}$ is stochastically independent of $\left(T_{(1)}, \ldots, T_{(n)}\right)$. Then, by using the total probability formula, the reliability function of $\delta$ can be decomposed into the form

$$
\bar{F}_{T}(t):=\mathrm{P}(T>t)=\sum_{k=1}^{n} s_{k} \mathrm{P}\left(T_{(k)}>t\right) .
$$

Equation (1) is at the basis of several applications of the concept of signature and, in particular, is used in the comparison of systems (see, e.g. [3], [4], [5], [6], [15], and [19, Chapters 4, 5]) and in the analysis of network reliability (see, e.g. [8] and [10]). Recently, it has been pointed out that the above formula can be suitably extended to the case when $T_{1}, \ldots, T_{n}$ are exchangeable; in this respect see, in particular, [11], [12], [14], [16], [17], and [21].

Let us now recall the combinatorial interpretation of signature. In the combinatorial approach, it is convenient to conceive that nonfailed components continue to work even if the system has already failed, so that we can record the subsequent failure times of all of the $n$ components and list them in the natural increasing ordering.

Denote by $\mathcal{P}_{n}$ the set of permutations of $\{1,2, \ldots, n\}$. For any $\pi \equiv(\pi(1), \pi(2), \ldots$, $\pi(n)) \in \mathcal{P}_{n}$, we can consider the case when the systems's components $C_{1}, \ldots, C_{n}$ fail according to the ordering $\pi$, i.e.

$$
T_{\pi(1)}<T_{\pi(2)}<\cdots<T_{\pi(n)} .
$$

We look at the instant of failure and determine the component that caused the system failure, setting $m(\pi):=k$ if $T=T_{(k)}$. Furthermore, for $k=1,2, \ldots, n$, set

$$
A_{k}:=\{\pi \in \mathcal{P} \mid m(\pi)=k\} .
$$

Thus, $\mathcal{A} \equiv\left(A_{1}, \ldots, A_{n}\right)$ is a partition of $\mathcal{P}_{n}$, and we have

$$
\sum_{k=1}^{n}\left|A_{k}\right|=\left|\mathcal{P}_{n}\right|=n !
$$

where $\left|A_{k}\right|$ denotes the cardinality of $A_{k}$. Taking into account the fact that $T_{1}, \ldots, T_{n}$ are i.i.d., it is easy to see that

$$
s_{k} \equiv \frac{\left|A_{k}\right|}{n !}, \quad k=1,2, \ldots, n .
$$

Finally, we recall here the notions of cumulative signature and tail signature. We set

$$
S_{h}:=\sum_{i=1}^{h} s_{i}, \quad \bar{S}_{h}:=\sum_{i=h+1}^{n} s_{i} .
$$

Definition 1. The vectors $S \equiv\left(S_{1}, \ldots, S_{n}\right)$ and $\overline{\boldsymbol{S}} \equiv\left(\bar{S}_{1}, \ldots, \bar{S}_{n}\right)$ will be called the cumulative signature and the tail signature, respectively. It is also convenient and conventional to set

$$
S_{0}:=0, \quad \bar{S}_{0}:=1, \quad \text { and } \quad \bar{S}_{n}:=0 .
$$

The vectors $\boldsymbol{S}$ and $\overline{\boldsymbol{S}}$ have a direct role, and a basic combinatorial meaning, in the frame of system reliability.

Let us fix our attention on $\overline{\boldsymbol{S}}$ (dual facts can be enunciated for $\boldsymbol{S}$ ). By taking into account the form of the marginal distribution of the order statistics of i.i.d. random variables and recalling (1), we can also write

$$
\bar{F}_{T}(t)=\sum_{k=1}^{n} s_{k} \sum_{i=0}^{k-1}\left(\begin{array}{l}
n \\
i
\end{array}\right)[F(t)]^{i}[\bar{F}(t)]^{n-i} .
$$


By interchanging the order between the summation indexes $k$ and $i$, the expression in (2) can also be written in the form

$$
\bar{F}_{T}(t)=\sum_{i=0}^{n-1}\left(\begin{array}{l}
n \\
i
\end{array}\right)[F(t)]^{i}[\bar{F}(t)]^{n-i}\left(\sum_{k=i+1}^{n} s_{k}\right),
$$

i.e.

$$
\bar{F}_{T}(t)=\sum_{i=0}^{n-1}\left(\begin{array}{l}
n \\
i
\end{array}\right) \bar{S}_{i}[F(t)]^{i}[\bar{F}(t)]^{n-i}
$$

As a 'dual' form of this formula, we can also write the following expression for $F_{T}(t)=$ $1-\bar{F}_{T}(t)$, the distribution function of the system's lifetime $T$ :

$$
F_{T}(t)=\sum_{j=1}^{n} S_{j}\left(\begin{array}{l}
n \\
j
\end{array}\right)[F(t)]^{j}[\bar{F}(t)]^{n-j}
$$

As noted in [2], the coefficients $\bar{S}_{i}(i=0, \ldots, n-1)$ have a direct combinatorial meaning of their own. Such a meaning can be obtained by simply comparing (3) with the expression of system reliability in the form of a homogeneous $p q$-polynomial based on the number of path sets of different orders. The product $\left(\begin{array}{c}n \\ i\end{array}\right) \bar{S}_{i}$ can be thus viewed as the number of system path sets of order $n-i$. See also [7], [19, p. 80], and [20], where the dual property of the coefficients $S_{j}$, in terms of cut sets, is considered.

\section{Series and parallel connection of two coherent blocks}

Here we consider two coherent systems (or blocks) $\delta^{\prime}$ and $\delta^{\prime \prime}: \delta^{\prime}$ has $n$ components $C_{1}^{\prime}, \ldots, C_{n}^{\prime}$ and signature $\boldsymbol{s}^{\prime}=\left(s_{1}^{\prime}, \ldots, s_{n}^{\prime}\right)$, and $\delta^{\prime \prime}$ has $m$ components $C_{1}^{\prime \prime}, \ldots, C_{m}^{\prime \prime}$ (all different from $\left.C_{1}^{\prime}, \ldots, C_{n}^{\prime}\right)$ and signature $\boldsymbol{s}^{\prime \prime}=\left(s_{1}^{\prime \prime}, \ldots, s_{m}^{\prime \prime}\right)$. The vectors $\boldsymbol{S}^{\prime}, \boldsymbol{S}^{\prime \prime}$ and $\overline{\boldsymbol{S}}^{\prime}, \overline{\boldsymbol{S}}^{\prime \prime}$ denote the cumulative signatures and the tail signatures of $\delta^{\prime}$ and $\delta^{\prime \prime}$, respectively.

Now we consider the system $\&$, formed with the components $C_{1}^{\prime}, \ldots, C_{n}^{\prime}, C_{1}^{\prime \prime}, \ldots, C_{m}^{\prime \prime}$ and obtained as a series connection of $\delta^{\prime}$ and $\delta^{\prime \prime}$. Our aim in what follows is to find the signature $\boldsymbol{s}=\left(s_{1}, \ldots, s_{n+m}\right)$ of the system $\&$.

We can actually obtain a direct formula for the computation of the tail signature $\bar{S}=$ $\left(\bar{S}_{1}, \ldots, \bar{S}_{n+m}\right)$ of $\&$, i.e. for the computation of

$$
\bar{S}_{r}:=\sum_{i=r+1}^{n+m} s_{i}, \quad r=1,2, \ldots, n+m-2
$$

(it is obvious that in the present case $s_{n+m}=0$ and so $\bar{S}_{n+m-1}=0$ ).

In this respect we have the following result.

Proposition 2. The components of $\overline{\boldsymbol{S}}$ can be computed from $\overline{\boldsymbol{S}}^{\prime}$ and $\overline{\boldsymbol{S}}^{\prime \prime}$ by means of the formula

$$
\bar{S}_{u}=\sum_{i=\max (0, u-m)}^{\min (n, u)} \frac{\left(\begin{array}{c}
n \\
i
\end{array}\right)\left(\begin{array}{c}
m \\
u-i
\end{array}\right)}{\left(\begin{array}{c}
n+m \\
u
\end{array}\right)} \bar{S}_{i}^{\prime} \bar{S}_{u-i}^{\prime \prime}, \quad u=1,2, \ldots, n+m-2 .
$$


Proof. As is clear from the arguments recalled in Section 2, it is convenient, for the computation of $\overline{\boldsymbol{S}}$, to assume that the lifetimes of the components $C_{1}^{\prime}, \ldots, C_{n}^{\prime}, C_{1}^{\prime \prime}, \ldots, C_{m}^{\prime \prime}$ are i.i.d., continuous random variables; we denote by $\bar{F}(t)$ the corresponding survival function (we shall see next that the choice of $\bar{F}(t)$ is completely inessential).

We can fix $t$ arbitrarily (also this choice is inessential) and set, for brevity's sake,

$$
\omega=F(t), \quad \bar{\omega}=\bar{F}(t) .
$$

Recall (3) for the reliability of a system in terms of its tail signature. For the reliability of our system $\delta$ (formed as a series of $\delta^{\prime}$ and $\delta^{\prime \prime}$ ), we can write and compare the following two formulae:

$$
\sum_{u=0}^{n+m-1}\left(\begin{array}{c}
n+m \\
u
\end{array}\right) \bar{S}_{u} \omega^{u} \bar{\omega}^{n+m-u}
$$

and

$$
\left(\sum_{i=0}^{n-1}\left(\begin{array}{l}
n \\
i
\end{array}\right) \bar{S}_{i}^{\prime} \omega^{i} \bar{\omega}^{n-i}\right)\left(\sum_{j=0}^{m-1}\left(\begin{array}{c}
m \\
j
\end{array}\right) \bar{S}_{j}^{\prime \prime} \omega^{j} \bar{\omega}^{m-j}\right) .
$$

Now note that (7) can be rewritten as

$$
\sum_{u=0}^{n+m-1} \sum_{i=\max (0, u-m)}^{\min (n, u)}\left(\begin{array}{l}
n \\
i
\end{array}\right)\left(\begin{array}{c}
m \\
u-i
\end{array}\right) \omega^{u} \bar{\omega}^{n+m-u} \bar{S}_{i}^{\prime} \bar{S}_{u-i}^{\prime \prime}
$$

and is valid for any arbitrary $0<\omega<1$. By comparing (6) and (8) term by term with respect to the monomials $\omega^{u} \bar{\omega}^{n+m-u}$, we obtain (5).

Concerning (5), it is interesting now to present a probabilistic interpretation, based on combinatorial arguments. Let us denote by $M^{\prime}$ the anchor of $\delta^{\prime}$, i.e. the (random) number of failures, among the components of $\delta^{\prime}$, needed to meet the failure of $\delta^{\prime}$ and, similarly, let $M^{\prime \prime}$ be the anchor of $\delta^{\prime \prime}$. Furthermore, let us consider the events

$$
E_{h}^{\prime}: \equiv\left(M^{\prime}=h\right), \quad h=1,2, \ldots, n, \quad E_{k}^{\prime \prime}: \equiv\left(M^{\prime \prime}=k\right), \quad k=1,2, \ldots, m .
$$

Thus, if we assume that the lifetimes of $C_{1}^{\prime}, \ldots, C_{n}^{\prime}, C_{1}^{\prime \prime}, \ldots, C_{m}^{\prime \prime}$ are i.i.d. random variables, we can interpret the signatures' components as

$$
s_{h}^{\prime}=\mathrm{P}\left(E_{h}^{\prime}\right), \quad s_{k}^{\prime \prime}=\mathrm{P}\left(E_{k}^{\prime \prime}\right),
$$

for $h=1,2, \ldots, n$ and $k=1,2, \ldots, m$. Furthermore,

$$
\bar{S}_{h}^{\prime}=\mathrm{P}\left(M^{\prime}>h\right), \quad \bar{S}_{k}^{\prime \prime}=\mathrm{P}\left(M^{\prime \prime}>k\right) .
$$

In view of the very definition of signature we have

- $n ! \bar{S}_{h}^{\prime}$ permutations of the elements $\left\{C_{1}^{\prime}, \ldots, C_{n}^{\prime}\right\}$ that give rise to $\left(M^{\prime}>h\right)$;

- $n ! \bar{S}_{h}^{\prime} /(n-h)$ ! ordered subsequences (of length $h$ ) of the elements $\left\{C_{1}^{\prime}, \ldots, C_{n}^{\prime}\right\}$ that give rise to $\left(M^{\prime}>h\right)$ (i.e. such that if any such subsequences happen for the first $h$ failures then $\delta^{\prime}$ survives the first $h$ failures). 
Similarly, for any $k$, we have $m ! \bar{S}_{k}^{\prime \prime} /(m-k)$ ! ordered subsequences (of length $k$ ) of the elements $\left\{C_{1}^{\prime \prime}, \ldots, C_{m}^{\prime \prime}\right\}$ that give rise to $\left(M^{\prime \prime}>k\right)$.

By mixing, in any order, any such subsequence pair we obtain a subsequence, of length $h+k$, of elements of $C_{1}^{\prime}, \ldots, C_{n}^{\prime}, C_{1}^{\prime \prime}, \ldots, C_{m}^{\prime \prime}$ such that the event $(M>h+k)$ is verified, where $M$ denotes the anchor of $\&$.

In conclusion we obtain

$$
\left(\begin{array}{c}
h+k \\
h
\end{array}\right) \frac{n ! \bar{S}_{h}^{\prime}}{(n-h) !} \frac{m ! \bar{S}_{k}^{\prime \prime}}{(m-k) !}
$$

subsequences, of length $h+k$, of elements of $C_{1}^{\prime}, \ldots, C_{n}^{\prime}, C_{1}^{\prime \prime}, \ldots, C_{m}^{\prime \prime}$ such that

(a) the event $(M>h+k)$ is verified;

(b) of the first $h+k$ failures for $\delta$, exactly $h$ are failures of elements of $\delta^{\prime}$.

Now, any such subsequence (of length $h+k)$ can be 'continued' in $(n+m-h-k)$ ! different ways. Thus, we obtain

$$
(n+m-h-k) !\left(\begin{array}{c}
h+k \\
h
\end{array}\right) \frac{n ! \bar{S}_{h}^{\prime}}{(n-h) !} \frac{m ! \bar{S}_{k}^{\prime \prime}}{(m-k) !}
$$

permutations of the elements $C_{1}^{\prime}, \ldots, C_{n}^{\prime}, C_{1}^{\prime \prime}, \ldots, C_{m}^{\prime \prime}$ such that conditions (a) and (b) hold. By letting $r=h+k$ and

$$
A_{h, r}:=(n+m-r) !\left(\begin{array}{l}
r \\
h
\end{array}\right) \frac{n ! \bar{S}_{h}^{\prime}}{(n-h) !} \frac{m ! \bar{S}_{r-h}^{\prime \prime}}{(m-r+h) !},
$$

we can also say that, for any fixed $r(r=1,2, \ldots, n+m-2)$, we have $A_{h, r}$ permutations of the elements $C_{1}^{\prime}, \ldots, C_{n}^{\prime}, C_{1}^{\prime \prime}, \ldots, C_{m}^{\prime \prime}$ such that the following conditions hold:

$\left(\mathrm{a}^{*}\right)$ the event $(M>r)$ is verified;

(b*) of the first $r$ failures for $\delta$, exactly $h$ are failures of elements of $\delta^{\prime}$.

We then have a total of $A_{h, r}$ permutations of the elements $C_{1}^{\prime}, \ldots, C_{n}^{\prime}, C_{1}^{\prime \prime}, \ldots, C_{m}^{\prime \prime}$ such that the event $(M>r)$ is verified. Then, in view of the very definition of signature for the system $\&$, we obtain, for $r=1,2, \ldots, n+m-2$,

$$
\bar{S}_{r}=\mathrm{P}(M>r)=\frac{1}{(n+m) !} \sum_{h=\max (0, r-m)}^{\min (n, r)} A_{h, r}=\sum_{h=\max (0, r-m)}^{\min (n, r)} \frac{\left(\begin{array}{c}
n \\
h
\end{array}\right)\left(\begin{array}{c}
m \\
r-h
\end{array}\right)}{\left(\begin{array}{c}
n+m \\
r
\end{array}\right)} \bar{S}_{h}^{\prime} \bar{S}_{r-h}^{\prime \prime} .
$$

Remark 1. From our knowledge of $\overline{\boldsymbol{S}}$, the signature $s$ of the system $\&$ can be obtained by applying the relation

$$
s_{k}=\bar{S}_{k-1}-\bar{S}_{k}, \quad k=1,2, \ldots, n+m-1 .
$$

Thus, we can also obtain from (5) a formula for the computation of $s$. For this formula, a suitable combinatorial interpretation could be given as well.

Remark 2. It can happen (see, e.g. the examples in [11] and [13]) that two different systems, say $\delta_{A}^{\prime}$ and $\delta_{B}^{\prime}$, of the same order $m$, share the same signature $\boldsymbol{s}^{\prime}$. Let $\delta^{\prime \prime}$ be a third system of order $n$, and consider the two systems $\delta_{A}$ and $\delta_{B}$ respectively obtained as a series of $\delta_{A}^{\prime}$ with $\delta^{\prime \prime}$ 
and as a series of $\wp_{B}^{\prime}$ with $\delta^{\prime \prime}$. We note in this respect that, as a consequence of Proposition 2, the signatures of $s_{A}$ and $\delta_{B}$ share the same signature as well. Thus, we see that the signature of a series connection of two systems depends only on the original signatures and does not really depend on the systems.

This argument, in particular, shows a method to construct several examples of pairs of systems (of the same order) that share the same signature, starting from one such pair.

We now consider the case of the system $\delta^{\text {(par) }}$ obtained by putting in parallel $\delta_{1}$ and $\delta_{2}$. In such a case, by suitably dualizing the arguments above, we can easily obtain the following result about the cumulative signature $S$ of $\delta^{\text {(par) }}$.

Proposition 3. The components of $\boldsymbol{S}^{(\mathrm{par})}$ can be computed from $\boldsymbol{S}^{\prime}$ and $\boldsymbol{S}^{\prime \prime}$ by means of the formula

$$
S_{r}=\sum_{h=\max (0, r-m)}^{\min (n, r)} \frac{\left(\begin{array}{c}
n \\
h
\end{array}\right)\left(\begin{array}{c}
m \\
r-h
\end{array}\right)}{\left(\begin{array}{c}
n+m \\
r
\end{array}\right)} S_{h}^{\prime} S_{r-h}^{\prime \prime}, \quad r=2, \ldots, n+m .
$$

Remark 3. In defining the concept of signature we tacitly assumed that all the components $C_{1}, \ldots, C_{n}$ are $u p$ at time $t=0$, whence they progressively fail. For some types of problem, it can be useful to think, dually, of the case when $C_{1}, \ldots, C_{n}$ are all down at time $t=0$ and then progressively start to work (become $u p$ ) at random times $W_{1}, \ldots, W_{n}$. This situation naturally leads to the introduction of the notion of a construction spectrum ( $\mathrm{C}^{\star}$-spectrum) $\boldsymbol{b}=\left(b_{1}, b_{2}, \ldots, b_{n}\right)$, where $b_{i}$ is the probability that the system gets $u p$ at the instant $W_{(i)}$. The equation

$$
b_{n-i+1}=s_{i}
$$

relates the $C^{\star}$-spectrum to the signature. See $[9$, Chapters 6,10$]$ (note that, in this monograph, the term destruction-spectrum ( $\mathrm{D}$-spectrum) has been used to denote the signature).

Let us now address the relationship between Propositions 2 and 3. Here we have chosen to prove Proposition 2 first and then just claim that the proof of Proposition 3 can be obtained by applying dual arguments. An alternative approach would be to prove Proposition 3 first and then directly obtain from it Proposition 2 by using the relationship between the signature and the $\mathrm{C}^{\star}$-spectrum given in (9).

We also note in this respect that a further, alternative, approach to proving Proposition 2 or Proposition 3 could be given by taking into account the relationship existing between the signature and the number of path sets or cut sets of the different orders.

\section{Signatures of recurrent systems}

A recurrent system $\&$ is a coherent system composed of $N$ identical coherent modules (see [1, Chapter 1]) that are assembled by means of a coherent organizing structure. Different modules share the same structure but they have no common components. Thus, if $N$ is the number of modules and $m$ is the number of elements in a module, then the whole system $s$ has $\mathrm{Nm}$ components. For example, an organizing structure might be a bridge and each of its five modules is a coherent system which, as a particular case, may copy the organizing structure itself. More technically, we can describe the structure function $\varphi_{\&}$ of $\delta$ as follows. Let $\psi$ (the module structure) and $\Phi$ (the organizing structure), with $\psi:\{0,1\}^{m} \rightarrow\{0,1\}$ and $\Phi:\{0,1\}^{N} \rightarrow\{0,1\}$, be two given coherent structure functions. Then

$$
\varphi_{s}\left(x_{1}^{(1)}, \ldots, x_{m}^{(1)} ; \ldots ; x_{1}^{(N)}, \ldots, x_{m}^{(N)}\right)=\Phi\left(\psi\left(x_{1}^{(1)}, \ldots, x_{m}^{(1)}\right) ; \ldots ; \psi\left(x_{1}^{(N)}, \ldots, x_{m}^{(N)}\right)\right) .
$$


For our purposes, we extend the definition of the recurrent system to the case when the different modules may have different structures, provided that they share an identical signature.

We suppose that we know the signature $s^{\mathrm{o}}=\left(s_{1}^{\mathrm{o}}, s_{2}^{\mathrm{o}}, \ldots, s_{N}^{\mathrm{o}}\right)$ of the organizing structure and the signature $s^{*}=\left(s_{1}^{*}, s_{2}^{*}, \ldots, s_{m}^{*}\right)$ of the modules. Our goal is to obtain the signature $\boldsymbol{s}=\left(s_{1}, s_{2}, \ldots, s_{N m}\right)$ of the whole system $\S$. To this end, we consider the cumulative signature $S^{*}$ and the tail signature $\bar{S}^{*}$ of the modules, the cumulative signature $S^{\text {o }}$ and the tail signature $\overline{\boldsymbol{S}}^{\text {o }}$ of the organizing structure, and finally the cumulative signature $\boldsymbol{S}$ and the tail signature $\overline{\boldsymbol{S}}$ of the whole system $\$$.

Furthermore, we assume that the lifetimes of the $\mathrm{Nm}$ components are i.i.d. random variables distributed according to a continuous distribution function $G(t)$ (as already explained, this assumption is innocuous as far as the computation of the signature is concerned).

We begin by rewriting (3) for $\delta$ when this is looked at as a system of its basic $\mathrm{Nm}$ components:

$$
\bar{F}_{T}(t)=\sum_{r=0}^{N m-1}\left(\begin{array}{c}
N m \\
r
\end{array}\right) \bar{S}_{r}[G(t)]^{r}[\bar{G}(t)]^{N m-r} .
$$

On the other hand, the modules can be considered to have (independent) identically distributed lifetimes, due to the identity of their signatures. Thus, we can also write

$$
\bar{F}_{T}(t)=\sum_{u=0}^{N-1}\left(\begin{array}{l}
N \\
u
\end{array}\right) \bar{S}_{u}^{\mathrm{o}}\left[F_{M}(t)\right]^{u}\left[\bar{F}_{M}(t)\right]^{N-u} .
$$

Here $F_{M}(t)$ and $\bar{F}_{M}(t)$ respectively denote the distribution function and the survival function of the i.i.d. lifetimes of the $N$ different modules; thus, by applying to each module (3) and (4) with $F(t)$ replaced by $G(t)$, we can write

$$
\begin{aligned}
& \bar{F}_{M}(t)=\sum_{i=0}^{m-1} \bar{S}_{i}^{*}\left(\begin{array}{c}
m \\
i
\end{array}\right)[G(t)]^{i}[\bar{G}(t)]^{m-i}, \\
& F_{M}(t)=\sum_{j=1}^{m} S_{j}^{*}\left(\begin{array}{c}
m \\
j
\end{array}\right)[G(t)]^{j}[\bar{G}(t)]^{m-j} .
\end{aligned}
$$

From (11), and setting $p=G(t)$ and $q=\bar{G}(t)$ for brevity, we obtain

$$
\begin{aligned}
\bar{F}_{T}(t) & =\sum_{u=0}^{N-1}\left(\begin{array}{l}
N \\
u
\end{array}\right) \bar{S}_{u}^{\mathrm{o}}\left[\sum_{j=1}^{m} S_{j}^{*}\left(\begin{array}{c}
m \\
j
\end{array}\right) p^{j} q^{m-j}\right]^{u}\left[\sum_{i=0}^{m-1} \bar{S}_{i}^{*}\left(\begin{array}{c}
m \\
i
\end{array}\right) p^{i} q^{m-i}\right]^{N-u} \\
& =\sum_{u=0}^{N-1}\left(\begin{array}{l}
N \\
u
\end{array}\right) \bar{S}_{u}^{\mathrm{o}}\left[\sum_{j=1}^{m} S_{j}^{*}\left(\begin{array}{c}
m \\
j
\end{array}\right) p^{j} q^{m-j}\right]^{u}\left[\sum_{h=1}^{m} \bar{S}_{m-h}^{*}\left(\begin{array}{c}
m \\
h
\end{array}\right) p^{m-h} q^{h}\right]^{N-u} .
\end{aligned}
$$

We note that, for a coherent system with i.i.d. component lifetimes, (3) and (4) express the reliability function, and the lifetime's distribution function, in the form of a homogeneous polynomial of degree $n$ in the two variables $F(t)$ and $\bar{F}(t)$.

Here we have the homogeneous polynomial in (11), of degree $N$, whose variables are $F_{M}(t)$ and $\bar{F}_{M}(t)$ given in (12) and (13), which can in turn be written as homogeneous polynomials of degree $m$ in the variables $p$ and $q$. Expression (14), which is obtained as a composition of 
(11) with (12) and (13), is again a homogeneous polynomial (of degree $\mathrm{Nm}$ ) in the variables $p$ and $q$. This fact corresponds to a basic property in the algebra of polynomials. Even though this closure property is rather trivially seen to be valid, it has an essential role in our present deductions: let us denote by $D_{1}, D_{2}, \ldots, D_{N m}$ the coefficients of the obtained polynomial of degree $N m$ :

$$
\bar{F}_{T}(t)=\sum_{r=0}^{N m-1} D_{r} p^{r} \bar{p}^{N m-r}
$$

Obviously, $D_{1}, D_{2}, \ldots, D_{N m}$ depend on $\overline{\boldsymbol{S}}^{\text {o }}$ and $\overline{\boldsymbol{S}}^{*}$; thus, we will also write

$$
D_{r}=D_{r}\left(\overline{\boldsymbol{S}}^{\mathrm{o}}, \overline{\boldsymbol{S}}^{*}\right), \quad r=1,2, \ldots, N m .
$$

By comparing (15) with (10) we are now ready to formulate the following result.

Proposition 4. The tail signature of the recurrent system is given by

$$
\bar{S}_{r}=\frac{D_{r}\left(\overline{\boldsymbol{S}}^{\mathrm{o}}, \overline{\boldsymbol{S}}^{*}\right)}{\left(\begin{array}{c}
N m \\
r
\end{array}\right)}, \quad r=1,2, \ldots, N m .
$$

In principle, the expression of $D_{r}$ in terms of $\overline{\boldsymbol{S}}^{\text {o }}$ and $\overline{\boldsymbol{S}}^{*}$ is not difficult to find. However, carrying out the calculation analytically is an extremely tiresome task even for a rather small system having in total, say, $4 \cdot 3=12$ elements. An example presented in the next section will show how this task can be carried out quite efficiently using MATHEMATICA ${ }^{\circledR}$ (see, e.g. [23]).

Remark 4. In the case when the organizing structure is a series system (i.e. when $\overline{\boldsymbol{S}}^{\mathrm{o}}=$ $(1,0, \ldots, 0)),(14)$ obviously reduces to

$$
\bar{F}_{T}(t)=\left[\bar{F}_{M}(t)\right]^{N}=\left[\sum_{h=0}^{m-1} \bar{S}_{h}^{*}\left(\begin{array}{c}
m \\
h
\end{array}\right) p^{h} q^{m-h}\right]^{N} .
$$

The coefficients $\bar{S}_{0}, \ldots, \bar{S}_{N m-1}$ can then be obtained by comparing (16) with the expression of the reliability of the recurrent system written in terms of its basic components (recall (10)). Namely, we can write

$$
\bar{F}_{T}(t)=\sum_{r=0}^{N m-1}\left(\begin{array}{c}
N m \\
r
\end{array}\right) \bar{S}_{r} p^{r} q^{N m-r}=\left[\sum_{h=0}^{m-1} \bar{S}_{h}^{*}\left(\begin{array}{c}
m \\
h
\end{array}\right) p^{h} q^{m-h}\right]^{N}
$$

We might thus obtain for $\bar{S}_{0}, \ldots, \bar{S}_{N m-1}$ an expression that directly generalizes, for the $N>2$ case, the formula

$$
\bar{S}_{u}=\sum_{i=\max (0, u-m)}^{\min (n, u)} \frac{\left(\begin{array}{c}
n \\
i
\end{array}\right)\left(\begin{array}{c}
m \\
u-i
\end{array}\right)}{\left(\begin{array}{c}
n+m \\
u
\end{array}\right)} \bar{S}_{i}^{*} \bar{S}_{u-i}^{*}, \quad u=1,2, \ldots, n+m-2,
$$

which, in the $N=2$ case, we obtain by substituting the condition $\overline{\boldsymbol{S}}^{\prime}=\overline{\boldsymbol{S}}^{\prime \prime}=\overline{\boldsymbol{S}}^{*}$ into (5). The cumulative signature of a recurrent system, formed with a parallel organizing structure, can be obtained by reasoning similarly. 
Concerning Proposition 4, the following comments can be useful.

Note that, by taking into account the relationship existing between the signature of a system and the number of path sets or cut sets of the different orders, we might interpret the claim in Proposition 4 as a relationship between, say, the number of path sets (of different orders) of the recurrent systems and the number of path sets (of different orders) of the modules and of the organizing structure.

As an extension of the concept of a recurrent system, we considered the case of independent, possibly different, modules that share the same signature (see also Remark 2). Consequently, all the modules have the same reliability, when the lifetimes of all the basic components are considered to be i.i.d. Our assumption, however, is definitely necessary for our arguments; in fact, when making the (inessential) hypothesis that the components are identically distributed, we simultaneously need the lifetimes of modules to be identically distributed (besides being independent).

\section{Examples}

In this section we analyze two different examples that can help us to illustrate both the meaning and the application of our results.

Example 1. Here we consider the case of a series of two (nonnecessarily similar) blocks with $n=m=3$ components. We then use (5), which now reads

$$
\begin{aligned}
& \bar{S}_{1}=\frac{\left(\begin{array}{l}
3 \\
0
\end{array}\right)\left(\begin{array}{l}
3 \\
1
\end{array}\right)}{\left(\begin{array}{l}
6 \\
1
\end{array}\right)} \bar{S}_{0}^{\prime} \bar{S}_{1}^{\prime \prime}+\frac{\left(\begin{array}{l}
3 \\
1
\end{array}\right)\left(\begin{array}{l}
3 \\
0
\end{array}\right)}{\left(\begin{array}{l}
6 \\
1
\end{array}\right)} \bar{S}_{1}^{\prime} \bar{S}_{0}^{\prime \prime}, \\
& \bar{S}_{2}=\frac{\left(\begin{array}{l}
3 \\
0
\end{array}\right)\left(\begin{array}{l}
3 \\
2
\end{array}\right)}{\left(\begin{array}{l}
6 \\
2
\end{array}\right)} \bar{S}_{0}^{\prime} \bar{S}_{2}^{\prime \prime}+\frac{\left(\begin{array}{l}
3 \\
1
\end{array}\right)\left(\begin{array}{l}
3 \\
1
\end{array}\right)}{\left(\begin{array}{l}
6 \\
2
\end{array}\right)} \bar{S}_{1}^{\prime} \bar{S}_{1}^{\prime \prime}+\frac{\left(\begin{array}{l}
3 \\
2
\end{array}\right)\left(\begin{array}{l}
3 \\
0
\end{array}\right)}{\left(\begin{array}{l}
6 \\
2
\end{array}\right)} \bar{S}_{2}^{\prime} \bar{S}_{0}^{\prime \prime}, \\
& \bar{S}_{3}=\frac{\left(\begin{array}{l}
3 \\
0
\end{array}\right)\left(\begin{array}{l}
3 \\
3
\end{array}\right)}{\left(\begin{array}{l}
6 \\
3
\end{array}\right)} \bar{S}_{0}^{\prime} \bar{S}_{3}^{\prime \prime}+\frac{\left(\begin{array}{l}
3 \\
1
\end{array}\right)\left(\begin{array}{l}
3 \\
2
\end{array}\right)}{\left(\begin{array}{l}
6 \\
3
\end{array}\right)} \bar{S}_{1}^{\prime} \bar{S}_{2}^{\prime \prime}+\frac{\left(\begin{array}{l}
3 \\
2
\end{array}\right)\left(\begin{array}{l}
3 \\
1
\end{array}\right)}{\left(\begin{array}{l}
6 \\
3
\end{array}\right)} \bar{S}_{2}^{\prime} \bar{S}_{1}^{\prime \prime}+\frac{\left(\begin{array}{l}
3 \\
3
\end{array}\right)\left(\begin{array}{l}
3 \\
0
\end{array}\right)}{\left(\begin{array}{l}
6 \\
3
\end{array}\right)} \bar{S}_{3}^{\prime} \bar{S}_{0}^{\prime \prime}, \\
& \bar{S}_{4}=\frac{\left(\begin{array}{l}
3 \\
1
\end{array}\right)\left(\begin{array}{l}
3 \\
3
\end{array}\right)}{\left(\begin{array}{l}
6 \\
4
\end{array}\right)} \bar{S}_{1}^{\prime} \bar{S}_{3}^{\prime \prime}+\frac{\left(\begin{array}{l}
3 \\
2
\end{array}\right)\left(\begin{array}{l}
3 \\
2
\end{array}\right)}{\left(\begin{array}{l}
6 \\
4
\end{array}\right)} \bar{S}_{2}^{\prime} \bar{S}_{2}^{\prime \prime}+\frac{\left(\begin{array}{l}
3 \\
3
\end{array}\right)\left(\begin{array}{l}
3 \\
1
\end{array}\right)}{\left(\begin{array}{l}
6 \\
4
\end{array}\right)} \bar{S}_{3}^{\prime} \bar{S}_{1}^{\prime \prime}, \\
& \bar{S}_{5}=\frac{\left(\begin{array}{l}
3 \\
2
\end{array}\right)\left(\begin{array}{l}
3 \\
3
\end{array}\right)}{\left(\begin{array}{l}
6 \\
5
\end{array}\right)} \bar{S}_{2}^{\prime} \bar{S}_{3}^{\prime \prime}+\frac{\left(\begin{array}{l}
3 \\
3
\end{array}\right)\left(\begin{array}{l}
3 \\
2
\end{array}\right)}{\left(\begin{array}{l}
6 \\
5
\end{array}\right)} \bar{S}_{3}^{\prime} \bar{S}_{2}^{\prime \prime} .
\end{aligned}
$$

In the special case when $\delta^{\prime}$ and $\delta^{\prime \prime}$ are both parallel systems (i.e. $S^{\prime}=(1,1,0)$ and $S^{\prime \prime}=$ $(1,1,0))$ the above formulae lead to the solution

$$
\left(1,1, \frac{9}{10}, \frac{6}{10}, 0,0\right)
$$

In fact, as is easy to check by hand, the signature of $\delta$ in this case is the vector

$$
\left(0,0, \frac{1}{10}, \frac{3}{10}, \frac{6}{10}, 0\right) \text {. }
$$

Let us consider the case when $\wp^{\prime}$ has the structure function

$$
x_{1} \wedge\left(x_{2} \vee x_{3}\right)
$$


and $f^{\prime \prime}$ has the structure function

$$
x_{1} \vee\left(x_{2} \wedge x_{3}\right) \text {. }
$$

In this case we have $\bar{S}^{\prime}=\left(\frac{2}{3}, 0,0\right)$ and $\bar{S}^{\prime \prime}=(1,0,0)$, and the above formulae lead to the solution

$$
\bar{S}=\left(\frac{5}{6}, \frac{4}{10}, 0,0,0,0\right)
$$

Also, in this case it is easy to compute the signature of $\delta$, given by

$$
s=\left(\frac{1}{6}, \frac{13}{30}, \frac{12}{30}, 0,0,0\right)
$$

and which is the signature vector corresponding to $\bar{S}$.

Example 2. (Five bridge modules organized into a bridge structure.) The cumulative signature of the bridge is

$$
S_{1}^{\mathrm{o}}=0, \quad S_{2}^{\mathrm{o}}=\frac{1}{5}, \quad S_{3}^{\mathrm{o}}=\frac{4}{5}, \quad S_{4}^{\mathrm{o}}=1, \quad S_{5}^{\mathrm{o}}=1 .
$$

Whence, using (4) and (5), it is easy to deduce that the bridge has two cut sets of size 2, eight cut sets of size 3 , five cut sets of size 4 , and one cut set of size 5 . Therefore, the bridge module's down probability $q^{\star}$ is

$$
q^{\star}=2 q^{2} p^{3}+8 q^{3} p^{2}+5 q^{4} p+q^{5} .
$$

In the bridge structure the number of path sets of size $j, j=2,3,4,5$, coincides with the number of cut sets of the same size $j$ and, therefore,

$$
p^{\star}=1-q^{\star}=2 p^{2} q^{3}+8 p^{3} q^{2}+5 p^{4} q+p^{5} .
$$

Below we provide the steps necessary for calculating, using MATHEMATICA operators (see, e.g. [23]), the signature of the whole system.

1. Write the down probability of the organizing structure as

$$
Q=2 a^{2} b^{3}+8 a^{3} b^{2}+5 a^{4} b+a^{5} .
$$

2. Set $a:=q$ and $b:=p$, and $\operatorname{Expand}[Q]$. This operation produces a $(q, p)$-polynomial of order $5 \cdot 5=25$.

3. Set $b=1$. This will produce a polynomial $W$ in $q$ with powers ranging from 1 to 25 . (The coefficients at $q, q^{2}$, and $q^{3}$ will be 0 since the min cut set is 4.)

4. Apply the operator CoeficientList $[W, \mathrm{q}]$ which will produce the list of the coefficients at $q^{z}, z=4, \ldots, 25$. Set $D(z):=\operatorname{Coef} f\left[q^{z}\right]$.

5. Calculate the system's cumulative signature as

$$
S_{z}=D(z) * \frac{z !(25-z) !}{25 !}, \quad z=1, \ldots, 25 .
$$

See Table 1 for the cumulative signatures of the recurrent bridge system. 
TABLE 1: The cumulative signatures of the recurrent bridge system.

\begin{tabular}{ll}
\hline$z$ & \multicolumn{1}{c}{$S_{z}$} \\
\hline 3 & 0 \\
4 & 0.000632 \\
5 & 0.003463 \\
6 & 0.011203 \\
7 & 0.027876 \\
8 & 0.058923 \\
9 & 0.110859 \\
10 & 0.189717 \\
11 & 0.297752 \\
12 & 0.429485 \\
13 & 0.570515 \\
14 & 0.702248 \\
15 & 0.810283 \\
16 & 0.889141 \\
17 & 0.941077 \\
18 & 0.972124 \\
19 & 0.988797 \\
20 & 0.996537 \\
21 & 0.999368 \\
22 & 1 \\
23 & 1 \\
24 & 1 \\
25 & 1 \\
\hline
\end{tabular}

\section{References}

[1] Barlow, R. E. and Proschan, F. (1975). Statistical Theory of Reliability, and Life Testing. Holt, Riehart and Wiston, New York.

[2] Boland, P. J. (2001). Signatures of indirect majority systems. J. Appl. Prob. 38, 597-603.

[3] Block, H., Dugas, M. R. and Samaniego, F. J. (2007). Characterizations of the relative behavior of two systems via properties of their signature vectors. In Advances in Distribution Theory, Order Statistics, and Inference, Birkhäuser, Boston, MA, pp. 279-289.

[4] Block, H. W., Dugas, M. R. and Samaniego, F. J. (2007). Signature-related results on system lifetimes. In Advances in Statistical Modeling and Inference, World Scientific, Hackensack, NJ, pp. 115-129.

[5] Boland, P. J. and Samaniego, F. J. (2004). Stochastic ordering results for consecutive $k$-out-of- $n$ : $F$ systems. IEEE Trans. Reliab. 53, 7-10.

[6] Boland, P. J. And SAmaniego, F. J. (2004). The signature of a coherent system and its applications in reliability. In Mathematical Reliability: an Expository Perspective, Kluwer, Boston, MA, pp. 3-30.

[7] Boland, P. J., Samaniego, F. J. and Vestrup, E. M. (2003). Linking dominations and signatures in network reliability theory. In Mathematical and Statistical Methods in Reliability (Trondheim, 2002), World Scientific, River Edge, NJ, pp. 89-103.

[8] Elperin, T., Gertsbakh, I. B. and Lomonosov, M. (1991). Estimation of network reliability using graph evolution models. IEEE Trans. Reliab. 40, 572-581.

[9] Gertsbakh, I. B. and Shpungin, Y. (2010). Models of Network Reliability. CRC Press, Boca Raton, FL.

[10] Harms, D. D., Kraetzl, M., Colbourn, C. J. and Devitt, S. J. (1995). Network Reliability: Experiments with a Symbolic Algebra Environment. CRC Press, Boca Raton, FL.

[11] Kochar, S., Mukerjee, H. and Samaniego, F. J. (1999). The "signature" of a coherent system and its application to comparisons among systems. Naval Res. Logistics 46, 507-523.

[12] Marichal, J.-L. and Mathonet, P. (2011). System signatures for dependent lifetimes: explicit expressions and interpretations. J. Multivariate Anal. 102, 931-936.

[13] Navarro, J. and Rubio, R. (2010). Computation of signatures of coherent systems with five components. Commun. Statist. Simul. Comput. 39, 68-84. 
[14] NaVARro, J. AND RyChLIK, T. (2007). Reliability and expectation bounds for coherent systems with exchangeable components. J. Multivariate Anal. 98, 102-113.

[15] Navarro, J. And Shaked, M. (2006). Hazard rate ordering of order statistics and systems. J. Appl. Prob. 43, 391-408.

[16] Navarro, J., Samaniego, F. J., Balakrishnan, N. and Bhattacharaya, D. (2008). On the application and extension of system signatures in engineering reliability. Naval Res. Logistics 55, 313-327.

[17] Navarro, J., Spizzichino, F. and Balakrishnan, N. (2010). Applications of average and projected systems to the study of coherent systems. J. Multivariate Anal. 101, 1471-1482.

[18] Samaniego, F. J. (1985). On closure of the IFR class under formation of coherent systems. IEEE Trans Reliab. 34, 69-72.

[19] Samaniego, F. J. (2007). System Signatures and Their Application in Engineering Reliability. Springer, New York.

[20] Satyanarayana, A. and Prabhakar, A. (1978). New topological formula and rapid algorithm for reliability analysis of complex networks. IEEE Trans. Reliab. 27, 82-100.

[21] Spizzichino, F. (2008). The role of signature and symmetrization for systems with non-exchangeable components. In Mathematical Modeling for Reliability, IOS, Amsterdam, pp. 138-148.

[22] Triantafyllou, I. S. and Koutras, M. V. (2008). On the signature of coherent systems and applications. Prob. Eng. Inf. Sci. 22, 19-35.

[23] Wolfram, S. (1991). MATHEMATICA: A System for doing Mathematics by Computer, 2nd edn. AddisonWesley, Redwood, CA. 